\title{
Optimal postoperative refraction for good unaided near and distance vision with monofocal intraocular lenses
}

\author{
J A Bradbury, J S Hillman, A Cassells-Brown
}

\begin{abstract}
Forty five eyes with up to 2 dioptres of myopic astigmatism and up to 1 dioptre sphere either plus or minus following cataract extraction and implantation of a monofocal intraocular lens were examined to assess their unaided visual acuities. Forty three percent were able to see $6 / 12$ and $N 8$, and $60 \%$ were able to see $6 / 12$ and $N 10$. Subjects with between 1 and 2 dioptres of myopic astigmatism and virtually no sphere were able to see $6 / 12$ and $\mathrm{N} 10$ in $82 \%$ of cases. This study confirms the benefits to both distance and near vision of myopic astigmatism as an alternative to multifocal intraocular lenses.
\end{abstract}

The advent of intraocular lenses has revolutionised the quality of vision obtained by patients following cataract extraction. This is however a fixed focus system and most patients require a spectacle reading lens for good near vision. Multifocal intraocular lenses have been developed to overcome this problem. It has been demonstrated, however, by a number of authors that some patients with monofocal intraocular lenses can attain both good distance and reading vision without the need for a spectacle correcting lens. ${ }^{1-3}$

In a previous study ${ }^{1}$ we found that patients with up to 2 dioptres of myopic astigmatism obtained a vision of $6 / 12$ and $N 8$ in $50 \%$ of cases. The myopic astigmatism in that study was artificially constructed by correcting the spherical element of the refraction, leaving the myopic astigmatic element uncorrected.

This study was undertaken to assess the

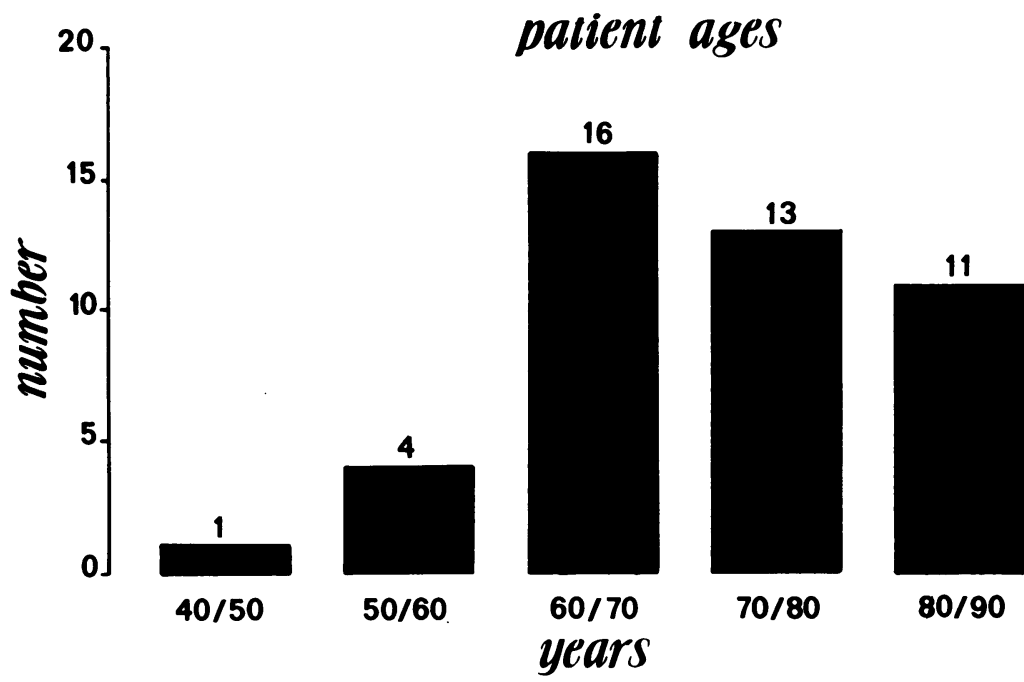

Figure 1 Age distribution of 45 patients following cataract extraction with implantation of an intraocular lens into the capsular bag. uncorrected visual acuities of subjects who had a best manifest corrected refraction of up to 2 dioptres of myopic astigmatism with up to 1 dioptre sphere either plus or minus.

\section{Materials and methods}

We studied 45 eyes in 45 patients after cataract extraction with implantation of a $J$ loop intraocular lens into the capsular bag. Patients were admitted consecutively to the study as they presented for routine review and were at least 3 months post cataract extraction. Patients with a corrected visual acuity of less than $6 / 12$ and N6 were excluded from the study.

All patients had preoperative biometry performed on a $3 \mathrm{M}$ Digiecho A scan biometer using the SRK Mk 2 formula and were calculated to obtain a spherical equivalent refraction of -1.5 dioptres postoperatively.

Postoperative keratometry was performed on a Haag-Streit Javal-Schiotz keratometer.

The following parameters were measured on each patient.

(1) Unaided and best corrected visual acuities at both near and distance. The distance acuity was measured at 6 metres using an illuminated Snellen chart, and the near at 0.3 metres with illumination from a $60 \mathrm{~W}$ light over one shoulder giving approximately $50 \mathrm{ft} \mathrm{lm}$

(2) Keratometry with either a Canon autokeratometer (18 eyes) or a Haag-Streit JavalSchiotz keratometer ( 27 eyes).

All refractions were transposed to a minus cylinder form. Statistical analysis was performed using the $\chi^{2}$ test.

All cylinders quoted are those obtained by best corrected manifest refraction unless stated otherwise.

\section{Results}

The 45 patients comprised 27 males and 18 females of mean age 73 years and age distribution as shown in Figure 1.

Table 1 Difference in dioptres between corneal astigmatism measured by keratometry and that determined by manifest refraction in 45 eyes after cataract extraction with implantation of a posterior chamber intraocular lens. (All cylinders in plus lens form)

\begin{tabular}{lcr}
\hline Keratometry minus refraction & Number & $\%$ \\
\hline$+3 \cdot 25$ to $+4 \cdot 0$ & 1 & 2 \\
$+2 \cdot 25$ to $+3 \cdot 0$ & 3 & 7 \\
$+1 \cdot 25$ to $+2 \cdot 0$ & 7 & 16 \\
zero to $+1 \cdot 0$ & 15 & 33 \\
zero & 5 & 11 \\
zero to $-1 \cdot 0$ & 11 & 25 \\
$-1 \cdot 25$ to $-2 \cdot 0$ & 3 & 7 \\
\hline
\end{tabular}


Table 2 Visual acuity levels attained with myopic $(-0 \cdot 5$ to $-1 \cdot 0)$, plus $(+0 \cdot 5$ to $+1 \cdot 0)$, and minimal sphere $(-0 \cdot 25$ to $+0 \cdot 25)$ with high and low amounts of myopic astigmatism

\begin{tabular}{lllll}
\hline Sphere & Cylinder & $6 / 12+N 8$ & $6 / 12+N 10$ & Below standard \\
\hline+0.5 to $+1 \cdot 0$ & $-1 \cdot 0$ to $-2 \cdot 0$ & $430 \%$ & $654 \%$ & $545 \%$ \\
+0.5 to $+1 \cdot 0$ & Zero to -0.75 & $133 \%$ & $266 \%$ & $133 \%$ \\
0.25 to -0.25 & $-1 \cdot 0$ to $-2 \cdot 0$ & $548 \%$ & $982 \%$ & $218 \%$ \\
0.25 to -0.25 & Zero to -0.75 & $250 \%$ & $250 \%$ & $250 \%$ \\
-0.5 to 1.0 & $-1 \cdot 0$ to $-2 \cdot 0$ & $646 \%$ & $754 \%$ & $646 \%$ \\
-0.5 to 1.0 & Zero to -0.75 & $266 \%$ & $266 \%$ & $133 \%$ \\
& & & & \\
\hline
\end{tabular}

Table 3 Difference in axis of astigmatism measured by keratometry and that measured by manifest refraction

\begin{tabular}{lrr}
\hline $\begin{array}{l}\text { Axis difference } \\
\text { (degrees) }\end{array}$ & No & $\%$ \\
\hline 0 to 5 & 10 & 23 \\
6 to 10 & 13 & 29 \\
11 to 15 & 9 & 20 \\
16 to 20 & 7 & 16 \\
21 to 25 & 0 & 0 \\
26 plus & 6 & 12 \\
& $\frac{1}{45}$ & $\frac{100}{}$ \\
\hline
\end{tabular}

$6 / 60$

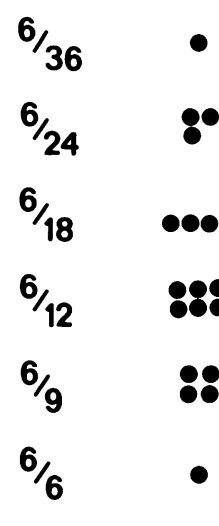

N6

N8

N10

The algebraic difference in dioptres between the corneal astigmatism measured by keratometry and that obtained by best corrected manifest refraction is shown in Table 1 . The astigmatism measured by keratometry was greater than that measured by manifest refraction in $58 \%$ of eyes. In only five $(11 \%)$ eyes did the astigmatism measured by keratometry equal that measured by manifest refraction. In $68 \%$ of eyes there was a difference of 1.0 dioptre or less, in $23 \%$ between 1.0 and 2.0 dioptres, and in $9 \%$ more than 2.0 dioptres with an overall difference of 0.88 dioptres.

Figure 2 Comparison of unaided distance and near acuities of 45 eyes after cataract extraction with implantation of an intraocular lens into the capsular bag.

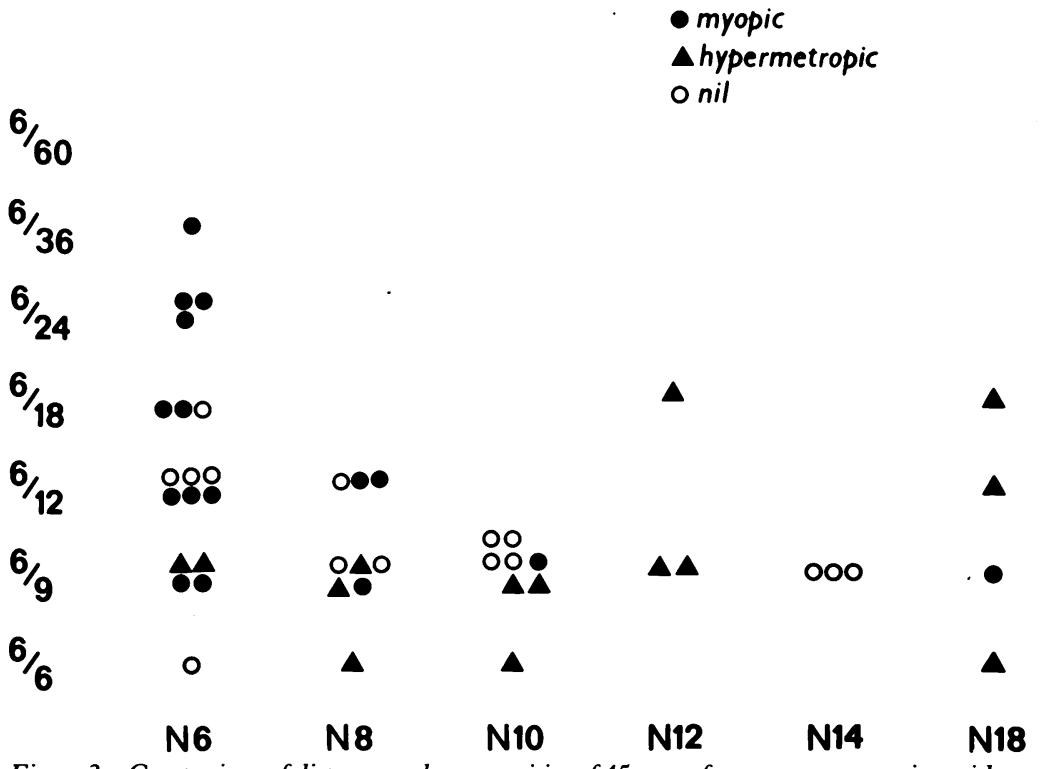

Figure 3 Comparison of distance and near acuities of 45 eyes after cataract extraction with implantation of an intraocular lens into the capsular bag and divided into those eyes with minus $(-0.5$ to $-1 \cdot 0)$, plus $(+0 \cdot 5$ to $+1 \cdot 0)$, and minimal $(-0.25$ to $+0 \cdot 25)$ sphere.
The uncorrected distance and near acuities of the eyes are shown in Figure 2 with $36(80 \%)$ achieving 6/12 or better, $27(60 \%)$ achieving N8 or better and 35 eyes (78\%) N10 or better. However considering both near and distance vision together 20 eyes (45\%) achieved $6 / 12$ and $\mathrm{N} 8$, and $28(62 \%)$ eyes achieved $6 / 12$ and $\mathrm{N} 10$.

Figure 3 shows the uncorrected visual acuities of the 45 eyes separated into plus spheres $(+0 \cdot 25$ to $+1 \cdot 0)$, minus spheres $(-0.25$ to $-1 \cdot 0)$, and minimal sphere $(+0.25$ to -0.25$)$. The spherical power refers to the spherical element of the best refraction lens form. In the myopic group $44 \%$ are below standard mainly because of poor distance vision, as expected near vision is very good. Below standard refers to an unaided visual acuity of less than $6 / 12$ and N10. In the hypermetropic patients $43 \%$ of the group are below standard mostly because of poor near vision, with a few failing for both near and distance. The group with minimal sphere performed best with only $27 \%$ being below standard. If we now divide these three groups into high and low amounts of myopic astigmatism (Table 2) the subgroup with minimal sphere and between 1 and 2 dioptres of myopic astigmatism sees best of all with $82 \%$ seeing at least $6 / 12$ and $\mathrm{N} 10$.

Statistical analysis of the subgroup with between 1 and 2 dioptres of myopic astigmatism and minimal sphere compared with the other subgroups produces a $p$ value of just more than the 0.05 value. This result is probably due to the low patient numbers in each subgroup.

\section{Discussion}

In our study $43 \%$ of eyes achieved $6 / 12$ and N8, and $60 \%$ achieved $6 / 12$ an N10. These figures are similar to those obtained in a previous study.'

The subgroup with between 1 and 2 dioptres of myopic astigmatism and minimal sphere achieved best vision with $82 \%$ seeing at least $6 / 12$ and N10. However a statistical analysis shows a $p$ value of just greater than $0.05\left(\chi^{2}\right)$ when compared with other subgroups within the study. Huber ${ }^{2}$ suggested a simple myopic astigmatism of up to 2 dioptres or a compound astigmatism of up to 3 dioptres allowed adequate near and distance vision, as did Datiles. ${ }^{3}$

Most authors believe the phenomenon of pseudophakic pseudoaccommodation to be due to myopic astigmatism, but have pointed out the value of a healthy reactive pupil in obtaining good unaided near and distance vision..$^{1-3}$ One author suggested that pupil size was more important than postoperative refraction. However, in a previous study ${ }^{1}$ we found pupil size to be insignificant. In that study $85 \%$ of eyes had an unaccommodated pupil size $2.5 \mathrm{~mm}$ or less and less than $2 \%$ had a pupil size greater than $4 \mathrm{~mm}$. All had mobile pupils, we feel that pupil size is unlikely to be significant within this group.

The astigmatism measured by best corrected manifest refraction and that by keratometry showed an average difference of 0.88 dioptres, with the value obtained by keratometry exceeding that obtained by refraction in $58 \%$ of cases and refraction by keratometry in $32 \%$ of cases. In $11 \%$ of cases keratometry equalled refraction. 
Some of this discrepancy may be explained by lenticular astigmatism combining with corneal astigmatism producing a change in power and axis as measured by manifest refraction, but this effect is likely to be small. Or in some cases the area of the cornea measured by keratometry may not be in the visual axis. (The difference in axis measured by astigmatism and that by manifest refraction is shown in Table 3.) The most obvious explanation of why the astigmatism measured by keratometry exceeds that measured by refraction in the majority of cases, is that the power of the astigmatism measured at the corneal plane will be greater than that measured at the spectacle plane.

The introduction of multifocal intraocular .lenses has generated much discussion on the relative merits of monofocal and multifocal lenses. Some authors have shown great benefits to patients in whom multifocal lenses have been implanted, others have been less enthusiastic. ${ }^{5}$ Multifocal lenses are more expensive, there is loss of contrast and reduced image quality, they require very accurate biometry and many designs are sensitive to decentration or pupil size. ${ }^{5}$ Monofocal lenses have relatively few problems and if an acceptable unaided distance and near vision is possible with monofocal lenses then that may offer an attractive alternative to implanting multifocal lenses.

A pure myopic astigmatism of 1.5 dioptres axis 180 degrees can be considered a desirable goal for postoperative refraction following cataract extraction and monofocal intraocular lens implantation. This can be achieved by calculating intraocular lens power for -1.5 dioptre sphere and controlling suture tension to give $a+1.5$ dioptre cylinder at axis 90 degrees. $^{6}$ This transposes to give $a-1.5$ dioptre cylinder axis 180 degrees.

1 Hillman JS, Bradbury JA. Apparent accommodation by myopic astigmatism with monofocal intraocular lenses. $E$ ur $\mathcal{f}$ Ref Surg 1990; 2: 101-4.

2 Huber C. Planned myopic astigmatism as a substitute for accommodation in pseudophakia. $\mathcal{F}$ Am Intraoc Implant Soc 1981; 7: 244-9.

3 Datiles MB, Gancayco T. Low myopia with low astigmatic correction gives cataract surgery patients good depth of focus. Ophthalmology 1990; 97: 922-6.

4 Nakazawa M, Ohtsuki K. Apparent accommodation in pseudophakic eyes after implantation of posterior chamber pseudophakic eyes after implantation of posterior chamber

5 Munton G. Bifocal intraocular lenses: Br f Ophthalmol 1991; 75: 367.

6 Hillman JS. Intraocular lens power calculation - the selection of formula. Trans Ophthalmol Soc UK 1985; 104: 693-8. 